This is a postprint version of the following published document:

Moreno, D., Wooders, J., (2017). Reserve prices in auctions with entry when the seller is risk-averse.Economics Letters, v. 154, pp. 6-9. Available in: https://doi.org/10.1016/j.econlet.2017.02.006

(C) Elsevier

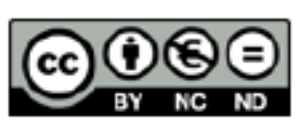

This work is licensed under a Creative Commons Attribution-NonCommercialNoDerivatives 4.0 International License. 


\title{
Reserve prices in auctions with entry when the seller is risk-averse
}

\author{
Diego Moreno ${ }^{\mathrm{a}, *}$, John Wooders ${ }^{\mathrm{b}}$ \\ a Departamento de Economía, Universidad Carlos III de Madrid, 28903, Getafe, Spain \\ ${ }^{\mathrm{b}}$ New York University Abu Dhabi, United Arab Emirates
}

\section{H I G H L I G H T S}

- We study public and secret reserve prices in auctions with endogenous entry.

- Seller's risk aversion raises the public reserve price, $r_{P}$, above the seller's cost, $c$, but lowers the secret reserve price, $r_{S}$, below the revenue maximizing reserve, $r_{0}$.

- Further, $r_{P}<r_{S}$.

- Hence, for a risk averse seller, reserve prices are ordered: $c<r_{P}<r_{S}<r_{0}$

\section{A B S T R A C T}

We show that risk aversion raises the public reserve price $r_{P}$ above the seller's cost $c$, but lowers the secret reserve price $r_{S}$ below the revenue maximizing reserve price $r_{0}$. Further, $r_{P}<r_{S}$. Hence, for a risk averse seller, public and secret reserve prices are ordered: $c<r_{P}<r_{S}<r_{0}$.

JEL classification:

D44

Keywords:

Second-price auctions

Endogenous entry

Public and secret reserve prices

Risk aversion

\section{Introduction}

Auction theory typically assumes that the seller is risk neutral. There are two good reasons for this assumption: First, sellers are often firms or government agencies whose objective is to maximize revenue. Second, risk neutrality facilitates the use of powerful methods from the mechanism design literature to characterize the optimal auction. The rise of the internet, however, has led to widespread consumer-to-consumer auctions on websites such as eBay. In these auctions, sellers are likely to be risk averse. Furthermore, the number of bidders is determined endogenously.

We study optimal public and secret reserve prices for risk averse sellers in second price auctions in which buyers simultaneously choose whether to enter the auction. Entering the auction

\footnotetext{
* Corresponding author. Fax: +34 916249329.

E-mail address: diego.moreno@uc3m.es (D. Moreno).
}

entails an entry cost, which we assume is the same for all buyers. A buyer who enters the auction observes her value for the object and then bids. The entry cost may be interpreted as the buyer's cost of learning his value for the item at auction. Buyers' values are private, and independently and identically distributed.

When the reserve price is public, that is, observed by buyers prior to making their entry decisions, the seller must take account of how the reserve price influences the buyers' entry decisions. Classic papers by McAfee and McMillan (1987) and Levin and Smith (1994) established that with a homogeneous entry costs, a reserve price equal to the seller's cost, $c$, is optimal for a risk neutral seller. We show that an optimal public reserve price for a risk averse seller, $r_{P}$, is above his cost, i.e., $c<r_{P}$.

When the reserve price is secret, the seller's choice of reserve price and buyers' entry decisions are effectively simultaneous. Hence the equilibrium reserve price maximizes the sellers' payoff given the bidders' entry decisions. For auctions with a fixed number of bidders, Hu et al. (2010) established that the optimal reserve price is independent of the number of bidders, and is 
smaller the more absolutely risk averse is the seller. An immediate implication of this result in our setting is that the secret reserve $r_{S}$ is independent of the bidders' entry decisions, and is smaller the more absolutely risk averse is the seller. In particular, when the seller is risk averse the secret reserve price is below the revenue maximizing reserve price $r_{0}$, i.e., $r_{S}<r_{0}$.

Taken together, these results establish that risk aversion has opposing effects on the optimal public and secret reserve prices: it raises public reserve prices, but lowers secret reserve prices. In addition, we show that if the seller is risk averse, an optimal public reserve price is smaller than the secret reserve price, i.e., $r_{P}<r_{S}$. Hence, for a risk averse seller, public and secret reserve prices are ordered: $c<r_{P}<r_{S}<r_{0}$.

\section{Model}

A single item is allocated using a second-price sealed-bid auction with a reserve price. There are $N \geq 2$ risk-neutral buyers who simultaneously decide whether to enter the auction. (While risk attitudes do not influence bids in a second price auction, they affect payoffs to entering the auction.) Entering the auction entails a cost $e>0$. Upon entering the auction, a buyer observes her value and then bids. Buyers' values are independently and identically distributed on an interval $[0, \omega]$ according to a distribution function $F$ with continuous density $f$, and increasing hazard rate $\lambda(x)=f(x) /[1-F(x)]$. The seller's cost of providing the item is $c \in[0, \omega)$.

Assuming that bidders follow their dominant strategy of bidding their value, if $n \geq 1$ bidders enter the auction and the reserve price is $r \in[c, \omega]$, then the payoff to an entering bidder is

$U(r, n)=\int_{r}^{\omega}\left(\int_{r}^{y} F(x)^{n-1} d x\right) f(y) d y$.

Clearly $U$ is decreasing in $r$ and $n$.

If all buyers enter the auction with the same probability $p$, the number of bidders in the auction follows a binomial distribution $B(N, p)$. Denote by $p_{n}^{N}(p)$ the binomial probability that there are exactly $n \in\{0,1, \ldots, N\}$ bidders in the auction. The expected payoff of a buyer who enters the auction when every other buyer enters the auction with probability $p$ is $\mathbb{U}(r, p)-e$, where

$\mathbb{U}(r, p)=\sum_{n=0}^{N-1} p_{n}^{N-1}(p) U(r, n+1)$.

Since $U$ is decreasing in $r$ so is $\mathbb{U}$. And since $U(r, n)$ is decreasing in $n$, and for $p^{\prime \prime}>p^{\prime}$ the binomial distribution $B\left(N-1, p^{\prime \prime}\right)$ first order stochastically dominates $B\left(N-1, p^{\prime}\right), \mathbb{U}$ is decreasing in $p$. The payoff of a buyer who does not enter the auction is zero.

In a symmetric equilibrium of the entry game, each buyer enters the auction with a probability $p^{*}$ that solves the problem

$$
\max _{p \in[0,1]} p\left(\mathbb{U}\left(r, p^{*}\right)-e\right) .
$$

Since $\mathbb{U}$ is decreasing in $p$, then $p^{*}=1$ whenever $\mathbb{U}(r, 1) \geq e$, and $p^{*}=0$ whenever $\mathbb{U}(r, 0) \leq e$, whereas $p^{*} \in(0,1)$ is the unique solution to the equation

$\mathbb{U}(r, p)-e=0$

otherwise. Denote by $\rho(r)$ the unique solution to (1). It is easy to see that $\rho^{\prime}(r)<0$ whenever $\rho(r) \in(0,1)$.

In order to rule out trivial cases where there is no entry or buyers enter with probability one, we assume that

$\mathbb{U}(c, 1)<e<\mathbb{U}(c, 0)$.

Thus, $\rho(r)<1$ for $r \in[c, \omega]$, and $\rho(r)>0$ for $r$ near $c$.
Denote by $v: \mathbb{R} \rightarrow \mathbb{R}$ the von Neumann-Morgenstern utility function representing the seller's preferences. We assume that $v$ is twice continuously differentiable, increasing and concave, and normalize $v$ so that $v(0)=0$ and $v^{\prime}(0)=1$. Thus, the utility function of a risk-neutral seller is $v(x)=x$. The seller's Arrow-Pratt measure of absolute risk aversion is $R(x):=$ $-v^{\prime \prime}(x) / v^{\prime}(x)$. Hence $R(x) \equiv 0$ when the seller is risk neutral.

In an auction with a reserve price $r \in[c, \omega]$ and $n$ bidders, the seller's expected utility is $V(r, 0)=0$, and for $n \geq 1$ it is

$$
\begin{aligned}
V(r, n)= & n(1-F(r)) F(r)^{n-1} v(r-c) \\
& +\int_{r}^{\omega} v(x-c) d G_{2}^{(n)}(x),
\end{aligned}
$$

where $G_{2}^{(n)}$ the c.d.f. of the second highest value. Clearly $V(r, n)$ is increasing in $n$. If the seller is risk neutral, then (2) identifies seller profit, which we write as $\pi(r, n)$.

The seller's expected utility when all buyers enter with the same probability $p$ is

$\mathbb{V}(r, p)=\sum_{n=0}^{N} p_{n}^{N}(p) V(r, n)$

Since $V(r, n)$ is increasing in $n$ and $B\left(N, p^{\prime \prime}\right)$ first order stochastically dominates $B\left(N, p^{\prime}\right)$ for $p^{\prime \prime}>p^{\prime}$, then $\mathbb{V}(r, p)$ is increasing in $p$. If the seller is risk neutral, (3) identifies seller profit when the reserve price is $r$ and each bidder enters with probability $p$, which we write as $\Pi(r, p)$.

\section{Results}

The reserve price is public when it is observed by buyers prior to making entry decisions. In this scenario, the seller and buyers face a dynamic game in which the seller first chooses the reserve price $r \in[c, \omega]$, and then buyers, upon observing $r$, simultaneously choose whether to enter. We focus on subgame perfect equilibria in which buyers follow symmetric strategies. In a subgame perfect equilibrium bidders enter according to $\rho$, and the public reserve price solves the problem

$\max _{r \in[c, \omega]} \mathbb{V}(r, \rho(r))$.

We say that $r$ is an optimal public reserve price if $(r, \rho)$ is a subgame perfect equilibrium of the auction.

A direct implication of Levin and Smith's (1994) Proposition 6 is that if the seller is risk neutral, then there is a unique optimal public reserve price, which is equal to the seller's cost, $c$.

Proposition 1 establishes that a risk averse seller sets a reserve price greater than a risk neutral seller. As its proof shows, a marginal increase of the reserve price above $c$ has two effects on the seller's expected utility: holding the entry probability fixed, it raises the seller's expected utility by the same amount whether the seller is risk neutral or risk averse; and by reducing entry, it lowers the seller's expected utility by a larger amount when the seller is risk neutral than when he is risk averse. Since the expected utility of the risk neutral seller is maximized at $r=c$, the total effect is zero for the risk neutral seller, but is positive for the risk averse seller.

Proposition 1. If the seller is risk averse, then an optimal public reserve price $r_{P}$ satisfies $r_{P}>c$. 
Proof. Differentiating (3) we may write

$$
\begin{aligned}
& \left.\frac{d \mathbb{V}(r, \rho(r))}{d r}\right|_{r=c}-\left.\frac{d \Pi(r, \rho(r))}{d r}\right|_{r=c} \\
& =\rho^{\prime}(c) \sum_{n=0}^{N} \frac{d p_{n}^{N}(p)}{d p}[V(c, n)-\pi(c, n)] \\
& \quad+\sum_{n=0}^{N} p_{n}^{N}(\rho(c))\left[\left.\frac{\partial V(r, n)}{\partial r}\right|_{r=c}-\left.\frac{\partial \pi(r, n)}{\partial r}\right|_{r=c}\right] .
\end{aligned}
$$

Since $v(0)=0$ and $v^{\prime}(0)=1$, differentiating Eq. (2) yields

$$
\left.\frac{\partial V(r, n)}{\partial r}\right|_{r=c}=n(1-F(c)) F(c)^{n-1},
$$

which is independent of the seller's utility function $v$. Therefore the second term in (4) is zero. As for the first term, we have

$$
\begin{aligned}
V(c, n)-\pi(c, n) & =-\int_{c}^{\omega}[x-c-v(x-c)] d G_{2}^{(n)}(x) \\
& =-\int_{0}^{\omega} \Theta(x) d G_{2}^{(n)}(x),
\end{aligned}
$$

where $\Theta(x):=0$ for $x \leq c$, and $\Theta(x):=x-c-v(x-c)$ for $x>c$. Thus, $\Theta^{\prime}(x)=0$ for $x \leq c$ and $\Theta^{\prime}(x)=1-v^{\prime}(x-c)>0$ for $x>c$ (because $v^{\prime}(0)=1$ and $v$ is concave), and therefore $\Theta$ is increasing. Furthermore, since $G_{2}^{(n+1)}(x)$ first order stochastic dominates $G_{2}^{(n)}(x), V(c, n)-\pi(c, n)$ is decreasing in $n$. Thus, since the binomial distribution $B\left(N, p^{\prime \prime}\right)$ first order stochastically dominates $B\left(N, p^{\prime}\right)$, whenever $p^{\prime \prime}>p^{\prime}$, and $V(c, n)-\pi(c, n)$ is decreasing in $n$, we have

$\sum_{n=0}^{N} p_{n}^{N}(p)[V(c, n)-\pi(c, n)]$

is decreasing in $p$. Since $\rho^{\prime}(c)<0$, and $\Pi(r, \rho(r))$ is maximized at $r=c$, i.e., $d \Pi(r, \rho(r)) /\left.d r\right|_{r=c}=0$, then

$$
\left.\frac{d \mathbb{V}(r, \rho(r))}{d r}\right|_{r=c}=\rho^{\prime}(c) \sum_{n=0}^{N} \frac{d p_{n}^{N}(p)}{d p}[V(c, n)-\pi(c, n)]>0,
$$

which establishes the proposition.

The reserve price is secret when it is observed by buyers only upon entering the auction, and is therefore unknown to the buyers when making entry decisions. In this scenario, the seller and buyers face a static game. A Nash equilibrium of this game is a pair $\left(r_{S}, p_{S}\right) \in[c, \omega] \times[0,1]$ such that $r_{S}$ solves the problem

$\max _{r \in[c, \omega]} \mathbb{V}\left(r, p_{S}\right)$,

and $p_{S}$ solves the problem

$\max _{p \in[0,1]} p\left(\mathbb{U}\left(r_{S}, p_{S}\right)-e\right)$.

Thus, if $\left(r_{S}, p_{S}\right)$ is a Nash equilibrium, then $p_{S}=\rho\left(r_{S}\right)$.

There are always trivial no-entry Nash equilibria in which the reserve price is high and buyers do not enter: a high reserve price is optimal when buyers enter with probability zero, and entering with probability zero is optimal when the reserve price is high. However, we focus on the (trembling hand) perfect equilibria, in which the reserve price is optimal for the seller when buyers enter with a vanishingly small probability.

By Proposition 5 in Hu et al. (2010), in a second price sealedbid auction with $n \geq 2$ bidders, the unique reserve price that maximizes the seller's expected utility, $V(\cdot, n)$, is the solution of the equation

$$
\Phi(r):=\frac{1}{\lambda(r)}-\frac{v(r-c)}{v^{\prime}(r-c)}=0,
$$

which is independent of $n$. Hence, for any $p>0$, the reserve price that uniquely maximizes $\mathbb{V}(\cdot, p)$ is the solution to Eq. (5). Moreover, by Theorem 2 in Hu et al. (2010), this reserve price is smaller the more absolute risk averse is the seller. Noting that these results hold when $n=1$ as well, Proposition 2 describes their implications for auctions with endogenous entry in which the reserve price is secret.

Proposition 2. When the reserve price is secret, there is a unique perfect equilibrium. In this equilibrium the reserve price $r_{S}$, which is the solution to Eq. (5), is independent of the number of buyers $N$, and is smaller the more absolute risk averse is the seller.

When the seller is risk neutral, the equilibrium secret reserve $r_{0}$ is the solution to the equation $r=c+1 / \lambda(r)$; hence $r_{0}>c$. Our theorem below describes the effects of seller risk aversion in auctions with public and secret reserve prices. Risk aversion has opposite effects when the reserve is public and when it is secret: it raises optimal public reserve prices above $c$, but lowers the secret reserve below $r_{0}$

Theorem. If the seller is risk averse, then an optimal public reserve price is below the secret reserve price, and hence $c<r_{P}<r_{S}<r_{0}$.

Proof. Let $r_{P}$ be any optimal public reserve price. First, we show that $r_{P} \leq r_{S}$. Let $r>r_{S}$. Since $r_{S}$ uniquely maximizes $V(\cdot, n)$ for all $n \geq 1$ (see the proof of Proposition 5 in Hu et al., 2010), then $V(r, n)<V\left(r_{S}, n\right)$ for all $n \geq 1$. Hence $\mathbb{V}(r, \rho(r))<$ $\mathbb{V}\left(r_{S}, \rho(r)\right)$. Also, since $\rho$ is decreasing, the binomial $B\left(N, \rho\left(r_{S}\right)\right)$ first order stochastically dominates $B(N, \rho(r))$; then $\mathbb{V}\left(r_{S}, \rho(r)\right) \leq$ $\mathbb{V}\left(r_{S}, \rho\left(r_{S}\right)\right)$. Therefore $\mathbb{V}(r, \rho(r))<\mathbb{V}\left(r_{S}, \rho\left(r_{S}\right)\right)$, and hence $r_{P} \leq$ $r_{S}$

Now we show that $r_{P} \neq r_{S}$. Since $\rho(r)>0$, and therefore $\mathbb{V}(r, \rho(r))>0$, for $r$ near $c$, then $\rho\left(r_{P}\right)>0$. Hence $r_{P}<r_{S}$ whenever $\rho\left(r_{S}\right)=0$. Suppose that $\rho\left(r_{S}\right)>0$. Then $\rho^{\prime}\left(r_{S}\right)<0$. Differentiating Eq. (3) yields

$$
\frac{d \mathbb{V}(r, \rho(r))}{d r}=\frac{\partial \mathbb{V}(r, p)}{\partial r}+\frac{\partial \mathbb{V}(r, p)}{\partial p} \rho^{\prime}(r)
$$

Again, since $r_{S}$ uniquely maximizes $V(\cdot, n)$, we have

$$
\left.\frac{\partial \mathbb{V}(r, p)}{\partial r}\right|_{r=r_{S}}=\left.\sum_{n=0}^{N} p_{n}^{N}(p) \frac{\partial V(r, n)}{\partial r}\right|_{r=r_{S}}=0 .
$$

Further, $\partial \mathbb{V}(r, p) / \partial p>0$ and $\rho^{\prime}\left(r_{S}\right)<0$ imply

$$
\left.\frac{\partial \mathbb{V}(r, p)}{\partial p}\right|_{r=r_{S}} \rho^{\prime}\left(r_{S}\right)<0 .
$$

Hence

$$
\left.\frac{d \mathbb{V}(r, \rho(r))}{d r}\right|_{r=r_{S}}<0,
$$

and therefore $r_{P} \neq r_{S}$.

Since $c<r_{P}$ by Proposition 1 , and $r_{S}<r_{0}$ by Proposition 2, then $c<r_{P}<r_{S}<r_{0}$.

An implication of our theorem is that profit is maximal when the reserve price is public and the seller is risk neutral. 


\section{Acknowledgments}

Moreno acknowledges financial support from the Ministerio Economía y Competitividad (Spain), grants ECO2014-55953-P and MDM2014-0431, and from the Comunidad de Madrid, grant S2015/HUM-3444. Wooders is grateful for financial support from the Australian Research Council's Discovery Projects funding scheme (project number DP140103566).

\section{References}

Hu, A., Matthews, S., Zou, L., 2010. Risk aversion and optimal reserve prices in firstand second-price auctions. J. Econom. Theory 145, 1188-1202.

Levin, D., Smith, J., 1994. Equilibrium in auctions with entry. Amer. Econ. Rev. 84, 585-599.

McAfee, P., McMillan, J., 1987. Auctions with entry. Econom. Lett. 23, 343-347. 